\title{
Review: major anxiety disorders all have substantive familial aggregation
}

Hettema JM, Neale MC, Kendler KS. A review and meta-analysis of the genetic epidemiology of anxiety disorders. Am J Psychiatry 2001 Oct;158:1568-78.

\section{QUESTION: In major anxiety disorders, what is the contribution of genetics and environment to their aetiology?}

\section{Data sources}

Studies were identified by searching Medline and by scanning the reference sections of identified studies and reviews.

\section{Study selection}

Studies were selected if they used operationalised diagnostic criteria, there was a systematic ascertainment of probands and relatives, a majority of participants were directly interviewed, the diagnostic assessment of relatives was blind to proband affection status, and for family studies there was a comparison group.

\section{Data extraction}

Data were extracted on study quality, patient characteristics, diagnostic criteria used, and outcome measures. A meta-analysis was used to estimate summary statistics of aggregate familial risk and heritability for each anxiety disorder.

\section{Main results}

5 family studies of panic disorder met the selection criteria. All 5 supported the familial aggregation of panic disorder. The summary odds ratio (OR) across the 5 studies was 5.0 (95\% CI 3.0 to 8.2$)$ showing a statistically significant association between panic disorder in probands and panic disorder in first degree relatives. 3 blinded twin studies of panic disorder met the selection criteria. These 3 studies supported a role for genetic factors in the aetiology of panic disorder yielding a heritability estimate of 0.48 (CI 0.41 to 0.54 ). 2 family studies of generalised anxiety disorder (GAD) met the selection criteria (1 was not blinded). The summary OR was 6.1 (CI 2.5 to 14.9 ) indicating an association between GAD in probands and their first degree relatives. 2 twin studies of GAD met the inclusion criteria. Combining the studies yielded a heritability estimate of 0.32 (CI 0.24 to 0.39 ). 4 family studies of phobias met the selection criteria. The summary OR across the studies was 4.1 (CI 2.7 to 6.1) which supports a familial risk for phobic disorders. 4 twin studies of phobias met the selection criteria. 1 large twin study supported that the major source of familial risk was genetic. 5 family studies of obsessive compulsive disorder (OCD) met the selection criteria (1 was not blinded). The summary OR across the 5 studies was 4.0 (CI 2.2 to 7.1). No twin studies on OCD met the selection criteria.

Sources of funding: NIMH Training Fellowship grants.

For correspondence: Dr K S Kendler, Virginia Institute for Psychiatric and

Behavioral Genetics,

Richmond, VA, USA.

kendler@hsc.vcu.edu

\section{Conclusions}

Panic disorder, generalised anxiety disorder (GAD), phobia, and obsessive compulsive disorder all have substantive familial aggregation. For panic disorder, GAD, and possibly phobias, genes largely explain this familial aggregation.

\section{COMMENTARY}

Hettema et al have shown support for a familial and genetic contribution to anxiety disorders. They note a high overall prevalence of anxiety disorders and substantial comorbidity with other mood disorders. Anxiety disorders have also been reported to be frequently comorbid with other medical conditions and to contribute to high utilisation of medical resources $^{1}$ and increased expense of health care. ${ }^{2}$ Children with a negative response to medical treatment, such as needle phobia, have poorer medical functioning and pain perception as adults, particularly after anxiety producing painful childhood medical experiences. ${ }^{3}{ }^{4}$

The findings of this meta-analysis are potent. There is a familial disposition toward a range of anxiety disorders and genetics substantially contribute to panic disorder, GAD, and probably phobias. This review is generally methodologically sound, with the limits of meta-analysis, the reliance on twin studies, and the uneven number of studies that met criteria for review, particularly for phobias and OCD.

How do clinicians interpret these data, which explain $30-40 \%$ of the variance leaving other non-specific factors to explain more than half of the total variance? Also, analyses were of anxiety disorders, and eliminated reports of anxiety symptoms. The data support screening for family history of anxiety disorder as a routine part of child and adult assessment, particularly if anxiety symptoms are part of the clinical presentation. If a history of familial anxiety disorder is revealed, it may support discussion of heritability as part of the clinical discussion with adults who have children.

However, we are still not adequately informed about the progression from risk to symptom to disorder. Why do some offspring of familial anxiety disorder develop similar disorders and some not? As the authors of this review point out, we need to understand the putative environmental risk factors that contribute to the activation of movement from risk to disorder, and how that should influence best treatment decisions.

\section{Rodger Kessler, $\mathrm{PhD}$ Central Vermont Medical Center Montpelier, Vermont, USA.}

1 Kawachi I, Sparrow D, Vokonas PS, et al. Symptoms of anxiety and risk of coronary disease. The Normative Aging Study. Circulation 1994;90:2225-9.

2 Katon W, Von Korff M, Lin E, et al. Distressed high utilizers of medical care. DSM-III-R diagnoses and treatment needs. Gen Hosp Psychiatry 1990;12:355-62.

3 Bachiocco V, Scesi M, Morselli AM, et al. Individual pain history and familial pain tolerance models: relationships to post-surgical pain. Clin J Pain 1993;9:266-71.

4 Lester N, Lefebvre JC, Keefe FJ. Pain in young adults: I. Relationship to gender and family pain history. Clin J Pain 1994;10:282-9. 